\title{
Assuring the Quality of the Course Learning Outcomes Assessment Process
}

\author{
Walid Ibrahim $^{1}$, Hoda Amer ${ }^{2}$ \\ ${ }^{1}$ Office Of Institutional Effectiveness, United Arab Emirates University, UAE, ${ }^{2}$ Department \\ Computer Science and Engineering, United Arab Emirates University, UAE.
}

\begin{abstract}
Learning Outcomes Assessment (LOA) provide educators with a practical instrument to review and enhance the alignment between the planned, delivered and experienced curriculum. Effective LOA processes help educators decide on the proper actions to take and the strategies to implement in order to ensure the continuous improvement of the student learning experience, and the attainment of the intended learning outcomes. Nonetheless, the adoption of LOA in higher education is still lagging and the assessment loop is rarely closed. This is mainly due to the indigent implementation of the assessment processes, and the vague definition of the responsibilities and quality assurance measures. This paper introduces a committee infrastructure to foster accountability and responsibility and assure the quality of the implemented assessment processes. The infrastructure has been established syccessfully over the last few years, and a noticeable improve in the execution of the assessment process has been detected.
\end{abstract}

Keywords: Learning outcomes assessment; quality assurance; committee; continuous improvement. 


\section{Introduction}

The last few decades witnessed a paradigm shift in higher education as more and more institutions have adopted the outcome-based education (OBE) model instead of the traditional curriculum-based one (Harden, 2007). The OBE model relies on the articulation of a set of learning outcomes that defines the knowledge, skills, and dispositions that students will have and able to demonstrate after successfully completing a learning experience (e.g., program, course, module, etc.). The defined learning outcomes are then used as guidelines for content development, instruction, and evaluation. They are also used to define the type and depth of learning students are expected to achieve, and provide a point of reference to assess the effectiveness of the learning experience. Moreover, they clearly communicate expectations to learners and prospective employers. This shift to the OBE model allows educators to focus more on what students know and are able to do by the time of graduation rather than the quality of the offered curriculum (i.e., output instead of input based).

To foster continuous improvements, the OBE model relies on assessing the learning outcomes defined for offered courses and programs through a periodic assessment cycle (Jankowski et al., 2018; Kuh et al., 2015). A typical learning outcomes assessment (LOA) process is shown in Figure 1. In order to have an effective assessment process, assessment tools should be selected carefully to collect data that represent the true students' attainment level (Keshavarz, 2011). Collected data from multiple tools across several semesters should be analysed thoroughly to identify positive and negative developing trends. The analysis possess should also identify deficiencies and areas for improvement, as well as the impact of implemented recommended actions from previous assessment cycles. Appropriate actions with implementation timeline should be recommended to remedy identified deficiencies. To close the assessment loop, the recommended actions are implemented during the subsequent offering and their impact is measured.

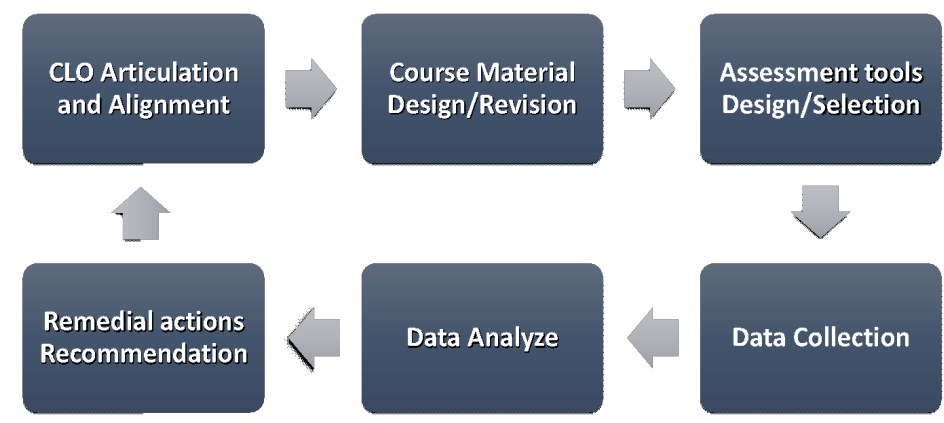

Figure 1. A Typical Course Learning Outcomes Assessment Process 
Despite the promising features of the outcomes-based model and its integrated assessment and continuous improvement cycle, its adoption in higher education is still lagging and the assessment loop is rarely closed (Kinzie et al., 2015; Kuh \& Ewell, 2010). This is mainly due to the indigent implementation of the assessment processes, and the vague definition of the responsibilities and quality assurance measures. This paper introduces the committee infrastructure established at (the University name is removed for blind review) to foster accountability and responsibility and assure the quality of the implemented assessment processes. The remaining of the paper is organized as follows. Potential LOA quality issues are discussed in Section 2, followed by the proposed quality assurance infrastrtute in Section 3 , and discussion remarks in Section 4.

\section{Potential issues that could affect the quality of the assessment process}

Although the typical LOA process shown in Figure 1 looks simple and straightforward to implement, in reality, the process is often lengthy and error prone. Therefore, having an integrated quality assurance framework that clearly defines the responsibility and accountability of each assessment task is essential for the successful implementation and closure of the assessment loop. For instance, the assessment process could define a task to thoroughly analyse the collected assessment data without clearly specifying who is responsible for completing the task and submitting the analysis remarks. In such a case, there is a considerable probability that no one will take the responsibility of getting the task done, which will cause the assessment process to terminate prematurely. Even if the task is eventually completed, it will be completed on voluntarily basis, which lacks the responsibility and accountability aspects necessary for quality assurance. The following subsections highlight some of the potential mistakes that could occur during the execution of the course assessment process and lead to the premature termination of the assessment loop.

\subsection{CLO Articulation and Alignment}

As shown in Figure 1, the course assessment process starts by articulating the intended course learning outcomes (CLOs) and aligning them with program learning outcomes and course objectives. Articulating appropriate CLOs is essential as they define the breadth and depth of the learning students are expected to achieve, and serve as guideline for content, instruction, and evaluation. The defined CLOs should be Specific, Measurable, Attainable, Relevant, and Time-bound (SMART). Each CLO must be articulated using specific language, and should start with appropriate Blooms Taxonomy action verb (Anderson et al., 2000) that defines its expected cognitive level.

Articulating appropriate CLOs is the initial step towards a successful execution of the assessment process. On the other hand, defining unmeasurable CLOs, or having a cognitive level mismatch between the CLOs and the course or the program level will cause the 
assessment process to fail. For instance, starting the CLO with vague action verbs such as "to know", "to appreciate", "to understand", "to be familiar with", etc. will make the CLO unmeasurable, as it does not clearly and explicitly define what is expected from students. The cognitive level of the CLO must be carfully selected to match the course and the program levels. For instance, the CLOs articulated for undergraduate freshmen courses should not have the same depth and breadth as a CLO of senior level courses, or CLOs articulated for Master or Doctorate level courses. Failing to articulate the CLO at the proper cognitive level is usually manifsated by the shortage of covering material and activities, which renders the CLO unmeasurable.

\subsection{Course Material Design and Revision}

The second task of the course assessment process is to define the course topics needed to cover the defined course learning outcomes. The aim of this task is to ensure that offered topics provide students with multiple opportunities to achieve the intended outcomes. It is also used to assure that the offered topics are aligned with the blooms cognitive level specified by the CLOs. Moreover, it is essential for closing the assessment loop, as it can be used to accurately identify the topics contributing to an unachieved CLO.

Failing to align the course topics and activities with the articulated CLOs is another cause of unmeaserable CLOs. In many cases, faculty are faced with a problem at the end of the semester that no assessment data is collected for one or more CLOs. This could be because the CLO was not covered at all; or it was covered, but was not assessed by any of the course activities. For instance, a course may define a CLO for students to communicate effectively in both oral and written format, however the course fails to define any topic or have any activity that covers communication skills. Failing to align course topics and activities with the cognitive level of the articulated CLOs will render the CLOs unmeasurable as well. For exmaple, a CLO could be articulated at the "Create" cognitive level (the highest level of Bloom's congnitive taxonomy), but the covered topics stops only at the "Apply" level.

\subsection{Assessment Tools Design and Selection}

The third task of the assessment process is to define the direct and indirect assessment tools that will be used to collect assessment data for each CLO. The aim is to select assessment tools that requires little extra time and effort, and do not overwhelm faculty and students with new tasks. Assessment data could be easily collected from regular learning activities (e.g. exams, portfolios, capstone projects, lab assignments, etc.) rather than additional tasks for students. The better the integration of the assessment tools into existing student work, the greater the probability that the assessment plans will succeed.

Collecting assessment data that does not truly represent the students' attainment level will lead to wrong analysis remarks and ineffective remedial actions. For instance, a final exam 
question might not be an appropriate tool to assess the student's ability to implement a design. Similarly, MCQs are not appropriate tools to measure the students' communication or information literacy skills. Therefore, the first step in the assessment plan is to identify the most appropriate assessment tools that will be used to assess each outcome. An appropriate assessment tool should be able to measure the competency addressed by the outcome effectively and accurately from multiple sections of the same course.

\subsection{Analyzing the Collected Data and Closing the Assessment loop}

Although closing the assessment loop is essential for continuous quality improvement, it is typically where the assessment efforts are disrupted (Hutchings et al., 2015). For instance, course instructors may terminate the assessment process after reporting the collected assessment results without providing any analysis remarks or recommend remedial actions. They could happen because they do not fully understand their role in the assessment process, and belive the assessment contribution ends after reporting the assessment data. It could also occur because they do not have enough information or the tools to analyse the students performance across multiple assessment cycles.

Even when remedial actions are recommended, there is usually no follow up to ensure that the recommended actions are implemented during the following course offering, especially when remedial actions are documented hard course portfolios (paper format) or in a soft format that is difficult to look up and extract. Consequently, the continuous improvement cycle is interrupted and no noticeable improvement is usually detected in the students' performance during the following assessment cycles. This usually led to faculty frustration and reluctance to participate in the assessment effort, as they do not see the benefit of the extra effort of collecting and reporting assessment data.

\section{Defining Responsibility and Accountability}

In a process that involves many contributors, clearly defining the entities responsible for each task or activity is essential for accountability and quality assurance. For instance, when the process assigns a general responsibility for faculty member to analyse the collected assessment data, there is a chance that no one will take responsibility for getting that task done. Therefore, effective assessment processes should clearly define the entities responsible for each assessment task, and the task timeline. This will allow the quality assurance team to follow up with the entities responsible for missing or incomplete tasks.

To assure proper implementation of the assessment process and the effective closure of the assessment loop, the role of the following entities are defined in the assessment process:

- Course Coordinator

- Course Committee 
- Department Assessment Committee (DAC)

- Department Curriculum Committee (DCC)

\subsection{Course Committee}

A course committee is routinely formed at the beginning of the semester for each courses offered by multiple instructors for multiple sections. The committee is chaired by the course coordinator and comprised of all instructors teaching the course that semester or taught the course recently. The course committee is responsible for developing and implementing the course assessment plan. The committee meets at the beginning of the semester to:

1. discuss and approve any modification to the course syllabus proposed by the course coordinator,

2. review the teaching materials, and decide on the delivery timeline and milestones including common midterm and final exams, if needed,

3. select the summative assessment tools to be used for each learning outcome, and

4. discuss the remedial actions recommended from previous offering, and decide on their implementation plans.

Member of the course committees are responsible for collecting the assessment data using the selected assessment tools, and implementing the approved remedial actions. They also collect evidence on the effectiveness of the implemented actions. At the end of the semester, the course committee meets again to analyse the learning outcomes attainment results, discuss the impact of the implemented remedial actions, and decide on the recommended actions for subsequent offering.

\subsection{Course Coordinator}

To strengthen course ownership, a course coordinator is appointed by the department chair for each offered course. The term of appointment of the course coordinator is four academic years. The performance of course coordinator is reviewed by the department chair in due time for a renewal or a replacement decision. The course coordinator responsibilities include:

1. Act as a liaison for course-related material (e.g. course syllabus, assessment reports, etc.), and chair the course committee in the case of multiple sections course.

2. Maintain the official course material.

3. Prepare the course portfolio each time the course is offered and upload it to the online course repository.

4. Organize and lead the initial meeting with the course committee during the first week of the semester to discuss the course offering plan.

5. Setup periodic follow up meetings with members of the courses committee to ensure homogeneous and synchronized progress of the course delivery across all offered sections of the course (lectures and labs). 
6. Coordinate the preparation of the unified midterm and final exams, and ensure their alignment with the course learning outcomes.

7. Oversee the implementation of the approved remedial actions

8. Follow up with the course committee on the implementation of the approved assessment tools and collection of the assessment data.

9. Organize and lead the end of the semester meeting with the course committee

10. Upload the course analysis remarks and new recommended actions to the LOA management system along with the implementation details and impact of the remedial actions implemented during the semester.

The appointed course coordinator should assume the responsibilities of the course committee if the course committee is not formed (e.g., a new course).

\subsection{Department Assessment Committee}

Department Assessment Committee (DAC) is a standing committee appointed by the department chair at the beginning of each academic year. The DLOAC oversees the execution of the course assessment process and ensures that the assessment data are collected and analysed as per the approved assessment timeline.

The DAC also plays a crucial role in closing the assessment loop. The scope of the course remedial actions could vary from a simple action that does not require any approval or the approval of the course committee, to more complex actions that might require the approval of the department, college, and university assessment committees. Some actions such as changing the course modality (e.g., face-to-face, blended, online) might also require the approval of the accrediting agency. Therefore, the role of the DAC is instrumental in following up with the involved entities to ensure that the remedial actions are implemented and the assessment loop is closed effectively.

\subsection{Department Curriculum Committee}

The department curriculum committee (DCC) is another standing committee appointed by the department chair at the beginning of each academic year. The curriculum committee is responsible for reviewing and approving any modification to the offered courses proposed by the course committee. The modifications include course description, topics, modality, and learning outcomes.

\section{Discussions}

To address the requirement of national and international accreditation agencies, a unified course learning outcomes assessment process was developed and implemented across the (University name is removed for blind review) since 2013. Although assessment data was 
regularly collected from all offered courses, the University quality assurance unit was deeply concerned that the assessment process usually stops after the assessment data is collected and the assessment loop is hardly closed. To address this issue, the terms of reference of the course coordinator and course committee were created and added to the University's Quality Assurance Framework. The terms of reference DAC was also revised to add a new responsibility to oversee the the execution of the course assessment process.

An online LOA management system was also designed and developed in house to streamline the developed assessment process. The system provides the department chairs, chairs of DLOAC, and course coordinators with detailed information regarding the status of the course assessment process, as well as executive dashboards to track the submission of the assessment data and the associated analysis remarks, the progress in the implementation of the recommended remedial actions, as well as the impact of the implemented actions.

A significant improvement in the execution of the assessment process and the closure of the assessment loop is evident since the introduction of the new quality assurance measures and the deployment of the online management system. Assessment statistics show that more than 5000 CLO were assessed during Spring 2020 in 1010 offered courses. Around 2000 remedial actions were also recommended by the course committees, out of which 378 are already implemented and closed in Fall 2020.

\section{References}

Anderson, L., Krathwohl, D., Airasian, P., Cruikshank, K., Mayer, R., Pintrich, P., Raths, J., \& Wittrock, M. (2000). Taxonomy for Learning, Teaching, and Assessing, A: A Revision of Bloom's Taxonomy of Educational Objectives, Abridged Edition (1st edition). Pearson.

Harden, R. M. (2007). Outcome-Based Education: The future is today. Medical Teacher.

Hutchings, P., Kinzie, J., \& Kuh, G. D. (2015). Evidence of student learning: What counts and what matters for improvement. In Using evidence of student learning to improve higher education (pp. 27-50). Jossey-Bass.

Jankowski, N. A., Timmer, J. D., Kinzie, J., \& Kuh, G. D. (2018). Assessment That Matters: Trending toward Practices That Document Authentic Student Learning. In National Institute for Learning Outcomes Assessment. National Institute for Learning Outcomes Assessment. https://eric.ed.gov/?id=ED590514

Keshavarz, M. (2011). Measuring Course Learning Outcomes. Journal of Learning Design, 4(4), 1-9.

Kinzie, J., Hutchings, P., \& Jankowski, N. A. (2015). Fostering greater use of assessment results: Principles for effective practice. In Using Evidence of Student Learning to Improve Higher Education (pp. 51-72). San Francisco, CA, USA: Jossey-Bass.

Kuh, G. D., \& Ewell, P. T. (2010). The State of Learning Outcomes Assessment in the United States. Higher Education Management and Policy, 22(1), 1-20. https://doi.org/10.1787/hemp-22-5ks5dlhqbfr1 
Kuh, G. D., Ikenberry, S. O., Jankowski, N. A., Cain, T. R., Ewell, P. T., Hutchings, P., \& Kinzie, J. (2015). Using Evidence of Student Learning to Improve Higher Education (1st edition). Jossey-Bass. 